\title{
Regulation of Triglyceride Metabolism II. Function of mitochondrial GPAT1 in the regulation of triacylglycerol biosynthesis and insulin action
}

\author{
Maria R. Gonzalez-Baró ${ }^{1}$, Tal M. Lewin ${ }^{2}$, and Rosalind A. Coleman ${ }^{2}$ \\ ${ }^{1}$ Instituto de Investigaciones Bioquímicas de La Plata (CONICET-UNLP), La Plata, Argentina \\ ${ }^{2}$ Departments of Nutrition and Pediatrics, University of North Carolina at Chapel Hill, Chapel Hill, \\ North Carolina
}

\begin{abstract}
GPAT1, one of four known glycerol-3-phosphate acyltransferase isoforms, is located on the mitochondrial outer membrane, allowing reciprocal regulation with carnitine palmitoyltransferase-1. GPAT1 is upregulated transcriptionally by insulin and SREBP-1c and downregulated acutely by AMP-activated protein kinase, consistent with a role in triacylglycerol synthesis. Knockout and overexpression studies suggest that GPAT1 is critical for the development of hepatic steatosis and that steatosis initiated by overexpression of GPAT1 causes hepatic, and perhaps also peripheral, insulin resistance. Future questions include the function of GPAT1 in relation to the other GPAT isoforms and whether the lipid intermediates synthesized by GPAT and downstream enzymes in the pathway of glycerolipid biosynthesis participate in intracellular signaling pathways.
\end{abstract}

\section{Keywords}

insulin resistance; diacylglycerol; lysophosphatidate; sterol regulatory element binding protein; hepatic steatosis

\begin{abstract}
Triacylglycerol (TAG) synthesis begins with the acylation of glycerol-3-phosphate to form lysophosphatidic acid (LPA) (Fig. 1), a rate-limiting step catalyzed by one of several isoforms of glycerol-3-phosphate acyltransferase (GPAT, EC 2.3.1.15). Historically, mammals were believed to have two different GPAT isoforms: 1 ) an endoplasmic reticulum isoform that can be inactivated by $N$-ethylmaleimide (NEM) and other sulfhydryl reagents, has no long-chain acyl-CoA-substrate preference, and comprises up to $90 \%$ of the total cellular GPAT activity, and 2) a mitochondrial isoform that is resistant to NEM inactivation and prefers to use saturated acyl-CoAs (4). With the identification of a second mitochondrial isoform that is sensitive to NEM (12), and at least two endoplasmic reticulum isoforms (Ref. 1; Nagle CA, Reue K, and Coleman RA, unpublished data), it now appears that this view was incomplete (Table 1).
\end{abstract}




\section{GPAT1 Is a Member of the pfam 01553 Family of Glycerolipid Acyltransferases}

GPAT1 is an intrinsic membrane protein, located in the outer mitochondrial membrane. Recombinant mouse and rat GPAT1 open reading frames encode proteins of 827 and 828 amino acids, respectively, with a high degree of homology. Consistent with the hydrophobicity analysis, protease-protection experiments and topography studies using epitope-tagged, recombinant GPAT1 show two transmembrane domains (aa 472-493 and aa 576-592) with both the $\mathrm{NH}_{2}$ and $\mathrm{COOH}$ termini facing the cytosol and a loop (aa 494-575) facing the intermembrane space (4). The active-site residues (motifs $I-I V$, Table 1) lie within the $\mathrm{NH}_{2}$ terminal domain, but mutagenesis studies suggest that both the loop and the $\mathrm{COOH}$-terminal domain are required for enzyme activity. The $\mathrm{COOH}$-terminal domain interacts physically with the $\mathrm{NH}_{2}$-terminal domain and probably contributes to substrate binding and catalysis (23).

Members of the pfam 01553 family of glycerolipid acyltransferases contain four conserved motifs (Table 1) that were first identified by a bioinformatics approach in 1997 (4).

Subsequently, mutagenesis of invariant residues in these motifs verified that these highly conserved regions were essential for the activity of Escherichia coli GPAT (PlsB), mouse GPAT1, and human dihydroxyacetone-phosphate acyltransferase (DHAPAT) (4). Residues important for catalysis are the invariant histidine and aspartate in motif I, the phenylalanine, glycine, and arginine in motif III, and the proline in motif IV (4). Amino acids important for binding glycerol-3-phosphate are the phenylalanine and arginine in motif II and the glutamate and serine in motif III (4). A diverse array of acyltransferases have been assigned to the pfam 01553 family, but biochemical activities have been verified only for GPAT1 and -2, lysophosphatidic acid acyltransferase (AGPAT)-1 and -2, DHAPAT, lysophosphatidylcholine acyltransferase, lysophosphatidylglycerol acyltransferase, and monolysocardiolipin acyltransferase $(2,4,19)$. Although AGPAT-6 and -8 were originally identified as lysophosphatidic acid acyltransferases, each has GPAT activity, and we will term them GPAT4 and GPAT3, respectively, in this review. Despite its homology to other lipid acyltransferases, tafazzin exhibits a transacylase activity (25). The amino acids important for the catalytic activity of most pfam 01553 family members have yet to be identified.

\section{Lysophosphatidate Is Transported From Mitochondria to Endoplasmic Reticulum for the Final Steps of Complex Lipid Synthesis}

The location of GPAT1 on the outer mitochondrial membrane is surprising because the enzymes that catalyze the final biosynthetic steps that form TAG and the glycerophospholipids are located in the endoplasmic reticulum membrane (4). Although GPAT1 and -2 are located in the mitochondrial outer membrane, their LPA product is clearly used for the synthesis of complex glycerolipids other than mitochondrial cardiolipin. Transport of LPA to the endoplasmic reticulum appears to be mediated in vitro by liver-fatty acid binding protein (LFABP) which can export mitochondrial LPA to microsomes to convert it to phosphatidic acid (PA) (4). In livers from L-FABP knockout mice, oleate incorporation into DAG and TAG is reduced, but the impact on LPA synthesis has not been measured (21).

\section{GPAT1 Controls Acyl-CoA Use at the Outer Mitochondrial Membrane}

Fatty acids are activated to acyl-CoAs on the outer mitochondrial membrane before entering either the glycerolipid biosynthetic pathway via GPAT1 or the catabolic $\beta$-oxidation pathway via carnitine palmitoyltransferase-1 (CPT1). Thus we hypothesized that GPAT1 and CPT1 might compete for the same long-chain acyl-CoA substrates and channel acyl-CoAs toward different pathways. This hypothesis was supported by the fact that fatty acid oxidation by rat hepatocytes is diminished when GPAT1 is overexpressed $(13,15)$, whereas in Fpat $^{-1-}$ mice 
fatty acids are partitioned away from TAG synthesis and toward oxidation and ketogenesis (9).

Partitioning of fatty acids to degradative vs. biosynthetic fates is regulated by the AMPactivated protein kinase (AMPK), a sensor of cellular energy availability (4). AMPK is active when energy stores are deficient or when energy demand is high and promotes catabolic processes that supply ATP while inhibiting biosynthetic processes that use ATP. Thus active AMPK inhibits acetyl-CoA carboxylase and decreases malonyl-CoA, a potent inhibitor of CPT1. The low concentration of malonyl-CoA allows enhanced CPT1 activity and increased $\beta$-oxidation. AMPK concomitantly decreases GPAT1 activity, although it is not known whether GPAT1 itself or an inhibitor is phosphorylated. Both an activator of AMPK, 5amino-4-imidazolecarboxamideriboside, and the purified recombinant AMPK itself inhibit GPAT1 activity by $30-40 \%$ in hepatocytes and in muscle, simultaneously increasing oxidative ${ }^{14} \mathrm{CO}_{2}$ production (4). Exercise, however, decreases AMPK concomitantly with mitochondrial GPAT activity in liver but not muscle (4). Thus the action of AMPK regulates the channeling of acyl-CoAs toward $\beta$-oxidation and away from glycerolipid biosynthesis in a reciprocal and cocoordinated manner.

GPAT1 contains several putative casein kinase (CK) sites, and when rat liver mitochondria are incubated with recombinant CK2, GPAT activity increases twofold, as does the phosphorylation of an $85-\mathrm{kDa}$ protein identified as GPAT1 $(4,22)$. The physiological significance of GPAT1 activation by CK2 is unknown. CK2, a cytosolic serine/threonine kinase involved in the cell cycle, neoplasia, and virus infection signaling, is a constitutively active kinase, and although it has hundreds of target proteins, only a few are metabolic enzymes (17). In both rat liver (22) and T-lymphocytes (6), CK2 activates GPAT1 50- 200\%. Because CK2 also activates rat liver CPT1 20-25\% and diminishes CPT1 inhibition by malonyl-CoA (11), CK2, unlike AMPK, would not appear to be a reciprocal regulator of fatty acid synthesis and degradation.

\section{GPAT1 Is Regulated in a Manner Consistent With a Role in Initiating TAG Synthesis}

Although the specific cellular functions of each GPAT isoform are not yet understood, strong experimental evidence supports the participation of GPAT1 in de novo TAG synthesis under conditions of dietary carbohydrate or fat excess. Adenovirus- and plasmid-mediated overexpression of GPAT1 in Chinese hamster ovary and HEK293 cells (4) and in primary rat hepatocytes $(13,15)$ increases TAG content and oleate incorporation into TAG and variably affects very-low-density lipoprotein (VLDL) secretion. In contrast, in Gpatl knockout mice, hepatic TAG mass is $40 \%$ lower than in wild-type mice (4).

Enzymes required for TAG synthesis are coordinately regulated through the counterregulatory hormones insulin and glucagon. Insulin enhances and both fasting and diabetic states reduce the activities of lipogenic enzymes, including GPAT1. Thus, in rat liver and adipose tissue, a 48-h fast decreases GPAT1 protein expression and activity $>30 \%$, and sucrose refeeding of previously fasted rats increases GPAT1 protein expression and activity more than twofold in liver (4). Consistent with nutritional regulation, in perfused rat liver, insulin increases GPAT1 activity $34 \%$ (4). In addition, streptozotocin-induced diabetes decreases GPAT1 activity in rat epididymal fat $60 \%$ and insulin administration restores GPAT activity (4).

\section{Changes in GPAT1 Activity Are Mediated Transcriptionally}

When previously fasted mice are refed a high-carbohydrate diet, hepatic Gpat1 mRNA levels increase $>20$-fold owing to enhanced transcription rates (4). This refeeding-induced increase 
in Gpat1 message is not observed in streptozotocin-diabetic mice unless insulin is administered. Insulin regulation of Gpatl is mediated by induction of sterol regulatory element binding protein-1 (Srebf-1) transcription, the main transcriptional regulator of Gpatl, and glucagon-mediated elevation of cAMP opposes the action of insulin (7). The mouse Gpat1 promoter region contains three sterol regulatory element binding protein (SREBP-1) consensus sites that are responsible for SREBP-1-mediated transactivation (4); thus ectopic expression of SREBP-1c in 3T3-L1 adipocytes causes Gpat1 mRNA expression to increase 6.7-fold.

Similarly, in Srebf-1 $a$ and - $1 c$ transgenic mice, the hepatic Gpat 1 message increases 10- and 3 -fold, respectively (4). Although LXR agonists have been reported to upregulate Gpat1, LXR does not directly transactivate Gpat 1 but rather upregulates Srebf-1c (4). Furthermore, although carbohydrate feeding increases Gpat $1 \mathrm{mRNA}$, upregulation proceeds through insulin-mediated transactivation of Srebf-1c rather than through the carbohydrate response element (3).

\section{GPAT1 Is Required for Normal Metabolism of Acyl-CoA and Its Absence Increases Fatty Acid Oxidation and Oxidative Stress}

Gpat1 $^{-1-}$ mice are healthy and breed normally, indicating that GPAT1 is not essential for embryogenesis, reproduction, or synthesis of milk TAG (4). GPAT1 contributes only $10 \%$ of total GPAT activity in every tissue in which it has been reported, except for liver where it provides $30-50 \%$ of total GPAT activity. Thus it is not surprising that the major phenotype observed in $\mathrm{Gpatl}^{-/-}$mice involves liver. Because AMPK reciprocally regulates GPAT1 and CPT1 (4), we expected that mice lacking GPAT1 would not be able to counterbalance CPT1 and would therefore increase their oxidation of fatty acids. This expectation was validated in mice fed a diet that was high in fat and sucrose. In Gpat1 $^{-1-}$ mice plasma $\beta$-hydroxybutyrate and acyl-carnitines (suggesting incomplete fatty acid oxidation) were twice as high as in controls, and hepatic mitochondrial HMG-CoA synthase mRNA was upregulated. In addition, hepatic acyl-CoA content increased threefold in ppat $^{-1-}$ fed a low-fat diet, demonstrating the importance of GPAT1 in metabolizing acyl-CoAs, even under conditions in which dietary fat is restricted.

In vitro studies have shown that GPAT1 prefers to esterify saturated fatty acyl-CoAs, particularly palmitoyl-CoA, at the $s n-1$ position of glycerol-3-phosphate (4). The $\mathrm{Fpatl}^{-/-}$ mouse confirmed that the enzyme functions similarly in vivo. In $\mathrm{Gpat1}^{-{ }^{-}-}$mice, less palmitate is present in hepatic TAG, phosphatidylcholine (PC), and phosphatidylethanolamine (PE). The $\mathrm{PE}$ and PC contain $~ 21 \%$ less palmitate and 30 and $64 \%$ more stearate and oleate, respectively, in the $s n-1$ position. Surprisingly, PE and PC contain $~ 40 \%$ more arachidonate at the $s n-2$ position, suggesting that fatty acid species at the $s n-1$ position control the fatty acid species that are esterified at the $s n-2$ position. Arachidonate is also 21 and $67 \%$ higher in mitochondrial $\mathrm{PE}$ and PC, respectively, from Gpat $^{-/-}$liver than wild-type controls, and 4-hydroxynonenal, a product of arachidonate peroxidation, is also increased (8).

These increases in arachidonate content and in fatty acid oxidation in $\mathrm{Gpatl}^{-/-}$mice result in a $20 \%$ increase in the rate of reactive oxygen species production, a marked increase in sensitivity to the induction of the mitochondrial permeability transition, and an increase in both oxidative stress-related hepatocyte apoptosis and in bromodeoxyuridine labeling (8). Thus, in the absence of GPAT1, excess fatty acid oxidation appears to stress the liver, but the resulting increase in apoptosis is balanced by an increase in hepatocyte proliferation. Such changes in cellular viability and proliferation could alter the risk for liver carcinogenesis.

\section{GPAT1 Mediates Hepatic Fatty Acid Content and Insulin Sensitivity}

Female $\mathrm{Gpat1}^{-1-}$ mice weigh less than controls and have reduced gonadal fat pad weights and lower hepatic TAG content, plasma TAG, and secretion of VLDL-TAG (4). After a high-fat 
diet for $3 \mathrm{wk}$, liver from $\mathrm{Gpat1}^{-/-}$mice contains markedly lower amounts of TAG and diacylglycerol (DAG) than wild-type mice. The Fpat $^{-/-}$liver is protected from hepatic insulin resistance during a hyperinsulinemic euglycemic clamp, possibly due to reduced DAGmediated activation of PKC $\varepsilon$ (20). Although hepatic acyl-CoA had previously been implicated in the pathogenesis of insulin resistance, compared with wild-type mice, $\mathrm{Gpat1}^{-{ }^{--}}$mice exhibit increased hepatic insulin sensitivity despite an almost twofold elevation in hepatic acyl-CoA content (20). The contribution of GPAT1 to insulin resistance is further highlighted by a study showing that a $90 \%$ adenovirus-mediated shRNA knockdown of hepatic NEM-resistant GPAT1 activity in $o b / o b$ mice reduces liver TAG and DAG content $40-50 \%$ and reduces plasma glucose and cholesterol concentrations 30-40\% (24). These effects suggest that inhibiting GPAT1 might ameliorate hepatic steatosis and insulin resistance.

Because GPAT1 is upregulated by SREBP-1c, we determined what would happen to Gpat $^{-/-}$mice fed diets high in sucrose and fat (from coconut oil) or low in sucrose and fat for 4 mo (9). Although hepatic TAG content is $60 \%$ lower in ppatl $^{-1-}$ mice than in control mice fed the high-fat and -sucrose diet, hyperinsulinemia and glucose intolerance are greater in the Gpat $^{-/-}$mice, and expression of the gluconeogenic genes glucose-6-phosphatase and PEPCK is not suppressed. These data are opposite those observed with $3 \mathrm{wk}$ of high-fat (safflower oil) feeding (20), possibly because of the length of time on the diets or differences in the fatty acid species fed.

The short-term feeding studies and data from GPAT1 overexpression in hepatocytes suggested that GPAT1 overexpression would result in a fatty liver. Hepatic steatosis is commonly associated with insulin resistance, but the proximate cause of insulin resistance is not well understood. It has been variously suggested that lipid intermediates or inflammation due to activated NF- $\mathrm{kB}$ may play a role. When an adenovirus construct is used to overexpress GPAT1 in mouse or rat liver, hepatic fatty acid oxidation diminishes, the livers become fatty, and plasma becomes hyperlipidemic (Ref. 14; Nagle CA, An J, Shiota M, Torres TP, Cline GW, Liu ZX, Wang S, Catlin RL, Shulman GI, Newgard CB, Coleman RA, unpublished observations). In mice treated with adenovirus-GPAT1, hepatic TAG increases 12-fold, and adipocyte differentiation-related protein and stearoyl-CoA desaturase- 1 expression are induced (14). Although body and fat pad weights are similar in rats that overexpress GPAT1 and control rats that express green fluorescent protein, a hyperinsulinemic-euglycemic clamp study shows that hepatic insulin resistance develops, with a glucose output that is 2.5 -fold higher than present in control mice (Nagle CA, An J, Shiota M, Torres TP, Cline GW, Liu ZX, Wang S, Catlin RL, Shulman GI, Newgard CB, Coleman RA, unpublished observations). NF- $\kappa B$ and its target genes, IL- $1 \beta$, and TNF- $\alpha$ are not affected, however, indicating that inflammation does not play a role in this model of hepatic insulin resistance. However, hepatic DAG and LPA are elevated, suggesting a role for these lipid metabolites in the development of hepatic insulin resistance. $\mathrm{PKC} \varepsilon$ is also activated, suggesting that insulin resistance might be a response to the elevated liver DAG. In addition, glycogen synthesis and the uptake of 2-deoxyglucose are reduced in skeletal muscle, suggesting that overexpression of GPAT1 in liver causes mild peripheral insulin resistance: the higher TAG content in skeletal muscle probably occurred because of the hyperlipidemia. These results indicate that an increase in de novo hepatic TAG synthesis can cause hepatic and systemic insulin resistance in the absence of obesity or a lipogenic diet.

\section{GPAT1 in Tissues Other Than Liver}

Few recent studies have examined the function of GPAT1 in tissues other than liver. In rat splenic T-lymphocytes and in Jurkat cells GPAT1 activity correlates positively with lymphocyte proliferation (5). In several other tissues GPAT1 protein, mRNA expression, and specific activity are discordant (4), suggesting that GPAT1 might be regulated 
posttranslationally. For example, GPAT1 protein expression is high in heart and adrenal gland, but the specific activity of NEM-resistant GPAT is relatively low in these tissues.

\section{Questions for the Future}

In pathways of intermediary metabolism such as glycolysis, glycogen synthesis, the urea cycle, and gluconeogenesis, it is unusual to find more than one isoform catalyzing any enzymatic step, so why do we need at least four GPAT isoforms? Is it because of the multiple glycerolipid products that are produced and the need to regulate different pathways independently? If this were true, it is odd that GPAT1 seems to be the only regulated isoform, at least in liver and adipose tissue. However, we know virtually nothing about the microsomal GPATs. Are the activities of these isoforms regulated or coordinated in any tissue? Are their LPA products destined for different fates? Does LPA synthesized at the outer mitochondrial membrane by GPAT1 enter into the same pathways as the LPA that is synthesized by GPAT- 3 and -4 on the endoplasmic reticulum? Questions of different fates would imply that protein-protein interactions exist to channel lipid intermediates toward or away from particular pathways.

Another major question is whether GPAT isoforms alter the production of lipid intermediates that participate in signaling pathways. For many years it has been assumed that the lipid signals LPA, PA, and DAG arise only from phospholipase-mediated hydrolysis of membrane phospholipids. Now studies of deficient or overexpressed GPAT1 are forcing us to rethink lipid signaling. For example, GPAT1 deficiency decreases hepatic DAG content and PKC $\varepsilon$ activation (20) whereas overexpression does the opposite (Nagle CA, An J, Shiota M, Torres TP, Cline GW, Liu ZX, Wang S, Catlin RL, Shulman GI, Newgard CB, Coleman RA, unpublished observations). These effects suggest that the DAG formed during glycerolipid synthesis is able to interact with and activate PKC at distant membranes. Furthermore, LPA, a ligand for PPAR $\gamma(16)$, is also diminished in liver from $\mathrm{Fpat1}^{-1-}$ mice and increases when GPAT1 is overexpressed. Could physiological changes in GPAT1 activity alter the effects of PPAR $\gamma$ on its target genes? Does GPAT1 (or the other GPAT isoforms) alter substrates such as acyl-CoA and glycerol-3-phosphate and/or products such as LPA, PA, and DAG, all of which are signaling molecules, and have effects on intracellular signaling or transcription? These complex questions demand further concerted study of the four GPAT isoforms.

\section{Acknowledgments}

\section{GRANTS}

This work was supported by grants from the National Institutes of Health: DK-56598, DK-59935, R03-TW06034, and P30 DK-56350.

\section{REFERENCES}

1. Cao J, Li JL, Li D, Tobin JF, Gimeno RE. Molecular identification of microsomal acyl-CoA:glycerol 3-phosphate acyltransferase, a key enzyme in de novo triacylglycerol synthesis. Proc Natl Acad Sci USA 2006;103:19695-19700. [PubMed: 17170135]

2. Cao J, Liu Y, Lockwood J, Burn P, Shi Y. A novel cardiolipin-remodeling pathway revealed by a gene encoding an endoplasmic reticulum-associated acyl-CoA:lysocardiolipin acyltransferase (ALCAT1) in mouse. J Biol Chem 2004;279:31727-31734. [PubMed: 15152008]

3. Cha JY, Repa JJ. The liver X receptor and hepatic lipogenesis: the carbohydrate-response element binding protein is a target gene of LXR. J Biol Chem 2006;282:743-751. [PubMed: 17107947]

4. Coleman RA, Lee DP. Enzymes of triacylglycerol synthesis and their regulation. Prog Lipid Res 2004;43:134-176. [PubMed: 14654091] 
5. Collison LW, Jolly CA. Phosphorylation regulates mitochondrial glycerol-3-phosphate-1 acyltransferase activity in T-lymphocytes. Biochim Biophys Acta 2006;1761:129-139. [PubMed: 16431156]

6. Collison LW, Kannan L, Onorato TM, Knudsen J, Haldar D, Jolly CA. Aging reduces glycerol-3phosphate acyltransferase activity in activated rat splenic T-lymphocytes. Biochim Biophys Acta 2005;1687:164-172. [PubMed: 15708364]

7. Eberle D, Hegarty B, Bossard P, Ferre P, Foufelle F. SREBP transcription factors: master regulators of lipid homeostasis. Biochimie 2004;86:839-848. [PubMed: 15589694]

8. Hammond LE, Albright CD, He L, Rusyn I, Watkins SW, Doughman SD, Lemasters JJ, Coleman RA. Increased oxidative stress is associated with balanced increases in hepatocyte apoptosis and proliferation in glycerol-3-phosphate acyltransferase-1 deficient mice. Exp Mol Pathol. In Press. doi: 10.1016/j.yexmp.2006.12.004.

9. Hammond LE, Neschen S, Romanelli AJ, Cline GW, Ilkayeva OR, Shulman GI, Muoio DM, Coleman RA. Mitochondrial glycerol-3-phosphate acyltransferase-1 is essential in liver for the metabolism of excess acyl-CoAs. J Biol Chem 2005;280:25629-25636. [PubMed: 15878874]

10. Harada N, Hara S, Yoshida M, Zenitani T, Mawatari K, Nakano M, Takahashi A, Hosaka T, Yoshimoto K, Nakaya Y. Molecular cloning of a murine glycerol-3-phosphate acyltransferase-like protein 1 (xGPAT1). Mol Cell Biochem. doi:10.1007/s11010-006-9321-5.

11. Kerner J, Distler AM, Minkler P, Parland W, Peterman SM, Hoppel CL. Phosphorylation of rat liver mitochondrial carnitine palmitoyltransferase-I: effect on the kinetic properties of the enzyme. J Biol Chem 2004;279:41104-41113. [PubMed: 15247243]

12. Lewin TM, Schwerbrock NMJ, Lee DP, Coleman RA. Identification of a new glycerol-3-phosphate acyltransferase isoenzyme, mtGPAT2, in mitochondria. J Biol Chem 2004;279:13488-13495. [PubMed: 14724270]

13. Lewin TM, Wang S, Nagle CA, Van Horn CG, Coleman RA. Mitochondrial glycerol-3-phosphate acyltransferase- 1 directs the metabolic fate of exogenous fatty acids in hepatocytes. Am J Physiol Endocrinol Metab 2005;288:E835-E844. [PubMed: 15598672]

14. Lindén D, William-Olsson L, Ahnmark A, Ekroos K, Hallberg C, Sjogren HP, Becker B, Svensson L, Clapham JC, Oscarsson J, Schreyer S. Liver-directed overexpression of mitochondrial glycerol-3phosphate acyltransferase results in hepatic steatosis, increased triacylglycerol secretion and reduced fatty acid oxidation. FASEB J 2006;20:434-443. [PubMed: 16507761]

15. Lindén D, William-Olsson L, Rhedin M, Asztély AK, Clapham JC, Schreyer S. Overexpression of mitochondrial glycerol-3-phosphate acyltransferase in rat hepatocytes leads to decreased fatty acid oxidation and increased glycerolipid biosynthesis. J Lipid Res 2004;45:1279-1288. [PubMed: 15102885]

16. McIntyre TM, Pontsler AV, Silva AR, St. Hilaire A, Xu Y, Hinshaw JC, Zimmerman GA, Hama K, Aoki J, Arai H, Prestwich GD. Identification of an intracellular receptor for lysophosphatidic acid (LPA): LPA is a transcellular PPAR agonist. Proc Natl Acad Sci USA 2003;100:131-136. [PubMed: 12502787]

17. Meggio F, Pinna LA. One-thousand-and-one substrates of protein kinase CK2? FASEB J 2003;17:349-368. [PubMed: 12631575]

19. Nakanishi H, Shindou H, Hishikawa D, Harayama T, Ogasawara R, Suwabe A, Taguchi R, Shimizu T. Cloning and characterization of mouse lung-type acyl-CoA:lysophosphatidylcholine acyltransferase 1 (LPCAT1). Expression in alveolar type II cells and possible involvement in surfactant production. J Biol Chem 2006;281:20140-20147. [PubMed: 16704971]

20. Neschen S, Morino K, Hammond LE, Zhang D, Liu ZX, Romanelli AJ, Cline GW, Pongratz RL, Zhang XM, Choi CS, Coleman RA, Shulman GI. Prevention of hepatic steatosis and hepatic insulin resistance in mitochondrial acyl-CoA:glycerol-sn-3-phosphate acyltransferase 1 knock out mice. Cell Metab 2005;2:55-65. [PubMed: 16054099]

21. Newberry EP, Xie Y, Kennedy S, Han X, Buhman KK, Luo J, Gross RW, Davidson NO. Decreased hepatic triglyceride accumulation and altered fatty acid uptake in mice with deletion of the liver fatty acid-binding protein gene. J Biol Chem 2003;278:51664-51672. [PubMed: 14534295] 
22. Onorato TM, Chakraborty S, Haldar D. Phosphorylation of rat liver mitochondrial glycerol-3phosphate acyltransferase by casein kinase 2. J Biol Chem 2005;280:19527-19534. [PubMed: 15778226]

23. Pellon-Maison M, Coleman RA, Gonzalez-Baró MR. The C-terminal region of mitochondrial glycerol 3-phosphate acyltransferase-1 interacts with the active site region and is required for activity. Arch Biochem Biophys 2006;450:157-166. [PubMed: 16620760]

24. Xu H, Wilcox D, Nguyen P, Voorbach M, Suhar T, Morgan SJ, An WF, Ge L, Green J, Wu Z, Gimeno RE, Reilly R, Jacobson PB, Collins CA, Landschulz K, Surowy T. Hepatic knockdown of mitochondrial GPAT1 in ob/ob mice improves metabolic profile. Biochem Biophys Res Commun 2006;349:439-448. [PubMed: 16935266]

25. Xu Y, Malhotra A, Ren M, Schlame M. The enzymatic function of tafazzin. J Biol Chem 2006;281:39217-39224. [PubMed: 17082194] 


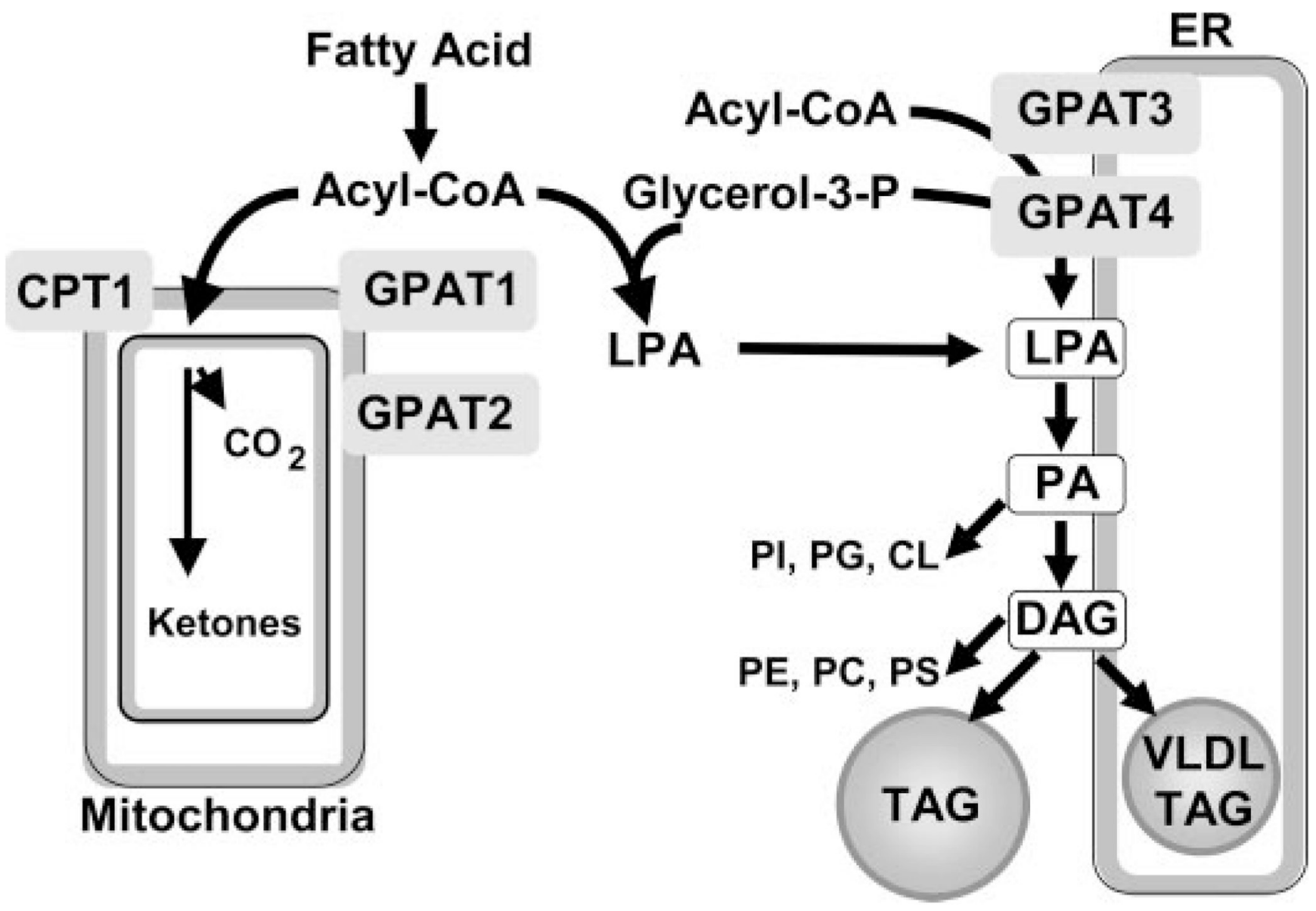

Fig. 1.

Triacylglycerol synthesis is initiated by four glycerol-3-phosphate acyltransferases (GPATs). GPAT1 and -2 are located on the outer mitochondrial membrane where they compete with carnitine palmitoyltransferase (CPT1) for acyl-CoAs. GPAT3 and 4 are located on the endoplasmic reticulum (ER). The lysophosphatidic acid (LPA) that these GPAT isoforms synthesize are acylated by lysophosphatidic acid acyltransferases to form phosphatidic acid (PA), the precursor for phosphatidylinositol (PI), phosphatidylglycerol (PG), and cardiolipin (CL). Lipin converts PA to diacylglycerol (DAG), the precursor of phosphatidylcholine (PC), phosphatidylethanolamine (PE), and phosphatidylserine (PS), and diacylglycerol acyltransferase acylates DAG to form triacylglycerol (TAG). VLDL, very-low-density lipoprotein. 


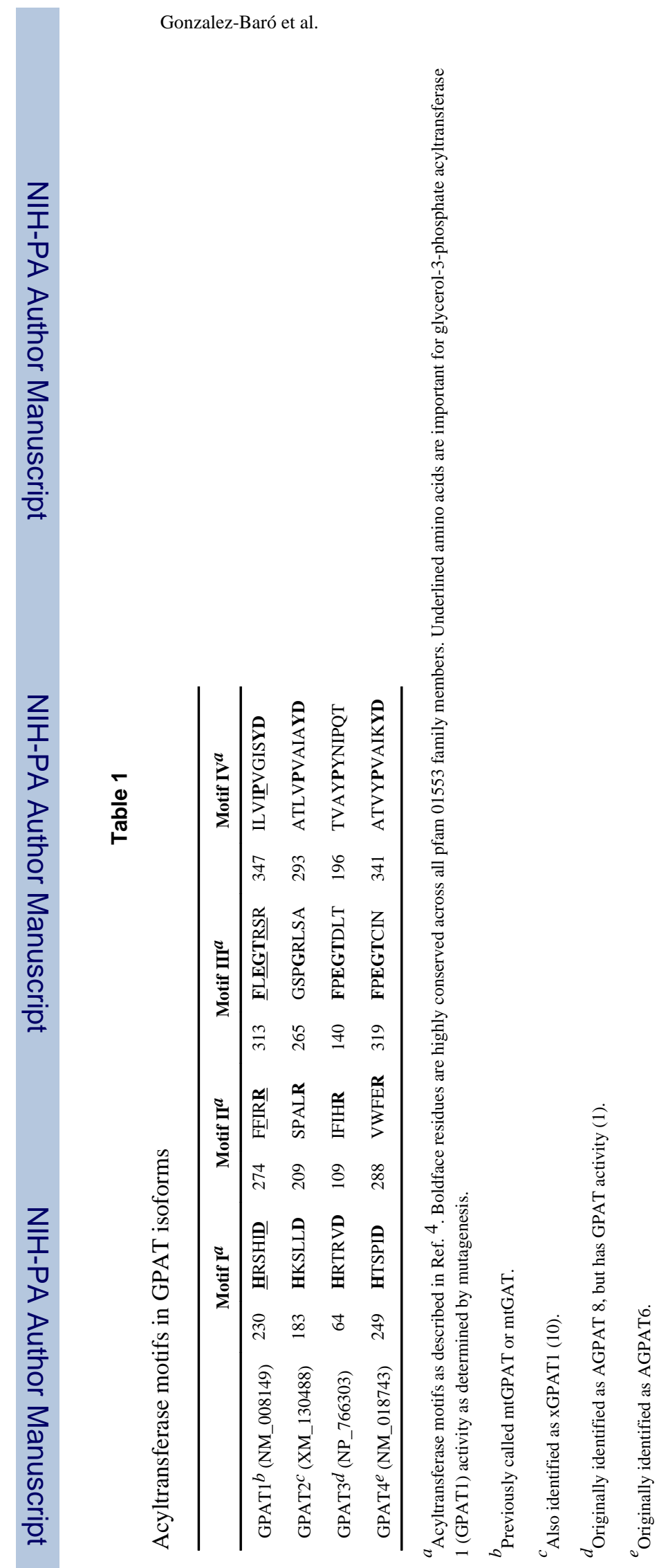

Am J Physiol Gastrointest Liver Physiol. Author manuscript; available in PMC 2010 February 10. 